\title{
Frecuencia de errores de los pacientes con su medicación
}

\author{
José Joaquín Mira, ${ }^{1}$ Isabel Maria Navarro, ${ }^{1}$ \\ Mercedes Guilabert ${ }^{1}$ y Jesús Aranaz ${ }^{2}$
}

Forma de citar

Mira JJ, Navarro IM, Guilabert M, Aranaz J. Frecuencia de errores de los pacientes con su medicación. Rev Panam Salud Publica. 2012;31(2):95-101.

RESUMEN Objetivo. Analizar la frecuencia de errores de medicación que son cometidos e informados por los pacientes.

Métodos. Estudio descriptivo basado en encuestas telefónicas a una muestra aleatoria de pacientes adultos del nivel primario de salud del sistema público español. Respondieron un total de 1247 pacientes (tasa de respuesta, 75\%). El 63\% eran mujeres y 29\% eran mayores de 70 años.

Resultados. Mientras 37 pacientes (3\%, IC 95\%:2-4) sufrieron complicaciones asociadas a la medicación en el curso del tratamiento, 241 (19,4\%, IC 95\%: 17-21) informaron haber cometido algún error con la medicación. Un menor tiempo de consulta $(\mathrm{P}<0,01)$ y una peor valoración de la información proporcionada por el médico $(\mathrm{P}<0,01)$ se asociaron al hecho de que en la dispensación en la farmacia le indicaran al paciente que el tratamiento prescrito no era apropiado.

Conclusiones. A los riesgos conocidos de sufrir un evento adverso, fruto de la intervención sanitaria por error del sistema o del profesional, hay que sumar los asociados a los errores de los pacientes en la autoadministración de la medicación. Los pacientes insatisfechos con la información proporcionada por el médico informaron un mayor número de errores.

Palabras clave Errores de medicación; cumplimiento de la medicación; participación del paciente; relaciones médico-paciente; atención primaria de salud; España.

La práctica clínica no está exenta de riesgos y son frecuentes los eventos adversos (EA) relacionados con la medicación o causados por problemas de comunicación entre el médico y el paciente (1-4).

Aunque la mayoría de los estudios de seguridad del paciente se han realizado en hospitales, en el nivel primario de salud se ha estimado una media de 11 EA por médico y año (5) y una tasa de errores de prescripción de 7,5\% $(6,7)$. El estudio APEAS (8), en España, identificó EA en 0,8\% (IC 95\%: 0,76 a 0,85) de las

\footnotetext{
1 Universidad Miguel Hernández de Elche, Elche, España. La correspondencia se debe enviar a José Joaquín Mira, jose.mira@umh.es

2 Hospital Universitari Sant Joan, Alicante, España.
}

consultas médicas, la mayoría evitables (64\%), aunque sólo 6\% tuvieron consecuencias graves. Los datos provenientes de países iberoamericanos son escasos y centrados en hospitales (4).

La duración de la consulta y el estilo de práctica clínica se relacionan con el riesgo de EA (9-11). A las consultas de atención primaria acuden pacientes con edad avanzada, múltiples patologías y polimedicados, circunstancias que se han relacionado con una mayor frecuencia de EA $(8,12)$. Cabe suponer, por tanto, que cualquier medida que contribuya a mejorar la seguridad de los pacientes en este nivel asistencial tendría un efecto positivo ya que afectaría a un amplio número de pacientes, que incluye a aquellos que presentan un mayor riesgo de presentar un EA.

Los estudios sobre EA en atención primaria son menos numerosos y se han centrado en la actuación de los profesionales. Sin embargo, la responsabilidad de la administración de la medicación reside en los pacientes una vez que están en su domicilio. Este aspecto ha sido escasamente estudiado, pero hay que considerar que los pacientes también cometen errores (equivocaciones por acción u omisión) que pueden conducir a EA $(13,14)$. Por ejemplo, hasta $88 \%$ de los pacientes diabéticos describen errores en la administración de insulina (15). 
Cabe esperar que, si mejora la información que proporciona el médico, se reducirán los riesgos para la seguridad de los pacientes a través de la disminución de sus errores con la medicación. Este estudio analiza estas cuestiones y la frecuencia de errores de los pacientes cuando se autoadministran la medicación en su casa.

\section{MATERIALES Y MÉTODOS}

Es un estudio descriptivo en el que se invitó a 1660 pacientes asistidos por médicos de atención primaria a contestar una encuesta telefónica, en la que se les preguntó acerca de los problemas experimentados en el curso del tratamiento debido a olvidos, confusiones o errores causados por ellos mismos en el último año, y su valoración del tratamiento y de la información que les proporcionaba su médico.

Este estudio se basó en resultados previos que habían puesto de manifiesto que los pacientes podían actuar como informadores suficientemente fiables de la frecuencia de incidentes para la seguridad de los pacientes (16-19).

Los pacientes se seleccionaron al azar mediante números aleatorios de entre el total de consultas de medicina general realizadas en siete centros de salud (dos ubicados en zonas rurales y cinco en zonas urbanas) del Sistema Público de la Comunidad Valenciana en España con más de tres años de implantación en su actividad comunitaria, durante el mes de abril de 2010. Como criterios de exclusión se consideraron: pacientes de pediatría; pacientes con enfermedades neurológicas o psiquiátricas; pacientes que hubieran presentado una queja, reclamación o denuncia en los últimos 6 meses (se consideró que la respuesta podría enmascarar la expectativa de compensación económica); y pacientes que rechazaran responder. El tamaño muestral se calculó para un error máximo del $3 \%$ para $P=Q=0,5$ y un nivel de confianza del $95 \%$.

La encuesta fue llevada a cabo por una empresa especializada en encuestas telefónicas mediante un sistema automatizado CATI (Computer Aided Telephone Interview) que garantiza el anonimato y el cumplimiento del plan de muestreo y mejora la fiabilidad de la codificación de las respuestas. Una vez seleccionado por el sistema el perfil del sujeto (considerando el sexo, la edad y el centro donde había sido atendido) se procedía a realizar la encuesta. En caso de falta de respuesta se realizaban intentos sucesivos de contacto telefónico (según las preferencias horarias indicadas por el paciente) hasta completar la encuesta. En caso de rechazo el sistema sustituía al paciente por otro con un perfil similar hasta completar el tamaño muestral. La encuesta se realizó de forma anónima, sin asociarse ningún dato personal a las respuestas y aplicando un protocolo de información y obtención de consentimiento para participar en este estudio conforme a las recomendaciones del Comité de Ética de la Universidad Miguel Hernández de España.

El contenido de la encuesta, de 12 ítems (ver anexo I), se diseñó considerando la experiencia del equipo investigador (Cuestionario de Percepción de Seguridad) (20) y los trabajos del Eurobarómetro (21), el Barómetro Sanitario Español (22), los estudios de Kuzel y cols. en EE.UU. (3), de Evans y cols. en Australia y Nueva Zelanda (23) y de Northcott y cols. en Canadá (24).

En la encuesta sobre EA se recurría a los términos "efectos indeseados o inesperados asociados al tratamiento" y "complicaciones" (mediante una pregunta indirecta). Estudios previos (25) han demostrado que estos términos son más apropiados y comprensibles para los pacientes que preguntar directamente por "eventos adversos". La frecuencia de errores relacionados con la medicación cometidos por el paciente se evaluó recurriendo a los términos "olvidos", "confusión" o "equívocos", por la misma razón. La encuesta incluía además una serie de preguntas (opciones de respuesta categorizadas en "bien", "regular" o "mal") para valorar la información proporcionada por el médico (valoración de la información, sensación de ser escuchado) y una pregunta sobre si se quedaba habitualmente con dudas tras la consulta. También se preguntó por la experiencia con la oficina de farmacia (a fin de determinar la frecuencia con la que se les indicaba a los pacientes que la prescripción probablemente no era apropiada en su caso). Para valorar la confianza en su médico se les preguntó si preferirían cambiar de médico. Por último, se analizaba si la respuesta al tratamiento era positiva y la satisfacción del paciente con el médico en una escala de 0 a 10 puntos.

Antes de realizar el estudio de campo se valoró el grado de comprensión de las preguntas y los tiempos de respuesta requeridos. Esta prueba piloto se realizó en 15 pacientes (que acudían a la consulta en centros de salud), y no motivó cambios relevantes.

El análisis de los datos de las respuestas al cuestionario se llevó a cabo mediante estadísticos descriptivos en una primera fase, en la que se analizó la calidad de los datos y se subsanaron posibles errores de codificación para, posteriormente, recurrir a los estadísticos bivariantes ji al cuadrado y prueba $t$. Cuando fue necesario, se segmentaron las respuestas en función del sexo y la edad (hasta 30, 31 a 64 y $\geq 65$ años). De forma convencional, se consideró que existían diferencias estadísticamente significativas cuando $P<0,05$.

\section{RESULTADOS}

Se analizaron las respuestas de 1247 pacientes (tasa de rechazo a contestar del 25\%). El error muestral fue de 2,7\%. La distribución de la muestra (cuadro 1) responde al perfil de pacientes habitual en las consultas de atención primaria.

De los pacientes encuestados, 37 (3\%, IC 95\%: 2 a 3,9) informaron alguna complicación o reacción inesperada en el último año relacionada con el tratamiento (cuadro 1). Conforme aumentaba la edad del informante la tendencia observada fue hacia el incremento de las complicaciones en el curso del tratamiento.

El 88,9\% de los encuestados valoró con siete o más puntos la satisfacción con su médico (media 8; desviación típica 1,3). No se hallaron diferencias en la satisfacción de los pacientes relacionadas con haber experimentado dificultades con el tratamiento indicado (prueba $t=0,01$; $P=0,9)$.

De los pacientes encuestados, 241 (19,3\%, IC 95\%: 17,1 a 21,5) informaron haber cometido olvidos o confusiones con la medicación en el último año. Los errores de los pacientes fueron tipificados del modo siguiente: en 175 casos (14\%; IC 95\%: 12,2 a 16) los pacientes dijeron olvidar con frecuencia las indicaciones del médico, 136 (11\%; IC 95\%: 9,2 a 12,6$)$ olvidaron tomar la medicación y 73 (6\%; IC 95\%: 4,5 a 7,2) confundieron los comprimidos (cuadro 1). En 151 pacientes $(12,1 \%)$ se detectaron tres errores, en $37(3 \%)$ dos y en $53(4,3 \%)$ solo uno. En todos los casos, contrariamente a lo esperado, los pacientes más jóvenes informaron un mayor número de errores que los pacientes de edad avanzada. 
CUADRO 1. Complicaciones y errores de los pacientes en el curso del tratamiento según el sexo y la edad, Comunidad Valenciana, España, 2010

\begin{tabular}{|c|c|c|c|c|c|c|c|c|c|c|c|c|}
\hline & \multirow[b]{2}{*}{$n$} & \multirow[b]{2}{*}{$\%$} & \multicolumn{2}{|c|}{$\begin{array}{c}\text { Efecto } \\
\text { inesperado o } \\
\text { complicación } \\
\text { en el último } \\
\text { año }\end{array}$} & \multicolumn{2}{|c|}{$\begin{array}{l}\text { Olvida con } \\
\text { frecuencia la } \\
\text { explicación } \\
\text { del médico }\end{array}$} & \multicolumn{2}{|c|}{$\begin{array}{l}\text { Olvida con } \\
\text { frecuencia } \\
\text { tomar la } \\
\text { medicación }\end{array}$} & \multicolumn{2}{|c|}{$\begin{array}{c}\text { Confunde } \\
\text { con frecuencia } \\
\text { los comprimidos }\end{array}$} & \multicolumn{2}{|c|}{$\begin{array}{l}\text { En la farmacia } \\
\text { alguna vez le } \\
\text { han indicado } \\
\text { que el } \\
\text { tratamiento } \\
\text { es inadecuado }\end{array}$} \\
\hline & & & No. & $\%$ & No. & $\%$ & No. & $\%$ & No. & $\%$ & No. & $\%$ \\
\hline \multicolumn{13}{|l|}{ Sexo } \\
\hline Hombres & 467 & 37,4 & 12 & 2,6 & 64 & 13,7 & 60 & 12,8 & 30 & 6,4 & 6 & 1,3 \\
\hline Mujeres & 780 & 62,6 & 25 & 3,2 & 111 & 14,2 & 76 & 9,7 & 43 & 5,5 & 7 & 0,9 \\
\hline \multicolumn{13}{|l|}{ Edad } \\
\hline$\leq 30$ & 135 & 10,8 & 2 & 1,5 & 30 & $22,2^{a}$ & 23 & $17^{a}$ & 16 & $11,9^{a}$ & 1 & 0,7 \\
\hline $31-64$ & 606 & 48,6 & 16 & 2,6 & 99 & 16,3 & 74 & 12,2 & 40 & 6,6 & 9 & 1,5 \\
\hline$\geq 65$ & 498 & 39,9 & 19 & 3,8 & 46 & 9,2 & 39 & 7,8 & 17 & 3,4 & 3 & 0,6 \\
\hline \multirow[t]{2}{*}{$\mathrm{NS} / \mathrm{NC}$} & 8 & 0,6 & & & & & & & & & & \\
\hline & 1247 & 100,0 & 37 & 3,0 & 175 & 14,0 & 136 & 10,9 & 73 & 5,9 & 13 & 1,1 \\
\hline
\end{tabular}

NS/NC: no sabe o no contesta.

a $P<0,001$.

En dos de los 37 (5,4\%; IC 95\%: 0 a 13) casos que experimentaron complicaciones con la medicación, los pacientes informaron haberse confundido de comprimidos (figura 1). Quienes dijeron que el tratamiento no tenía el efecto positivo esperado informaron en mayor proporción errores por acción (confundir los comprimidos, 16,7 frente a $4,9 \%, P=$ $0,04)$ o por omisión (olvido de la medicación, $22,2 \%$ frente a $8,5 \%, P=0,05)$.

FIGURA 1. Número de pacientes que informaron errores al administrarse la medicación en su casa según la encuesta telefónica, Comunidad Valenciana, España, 2010
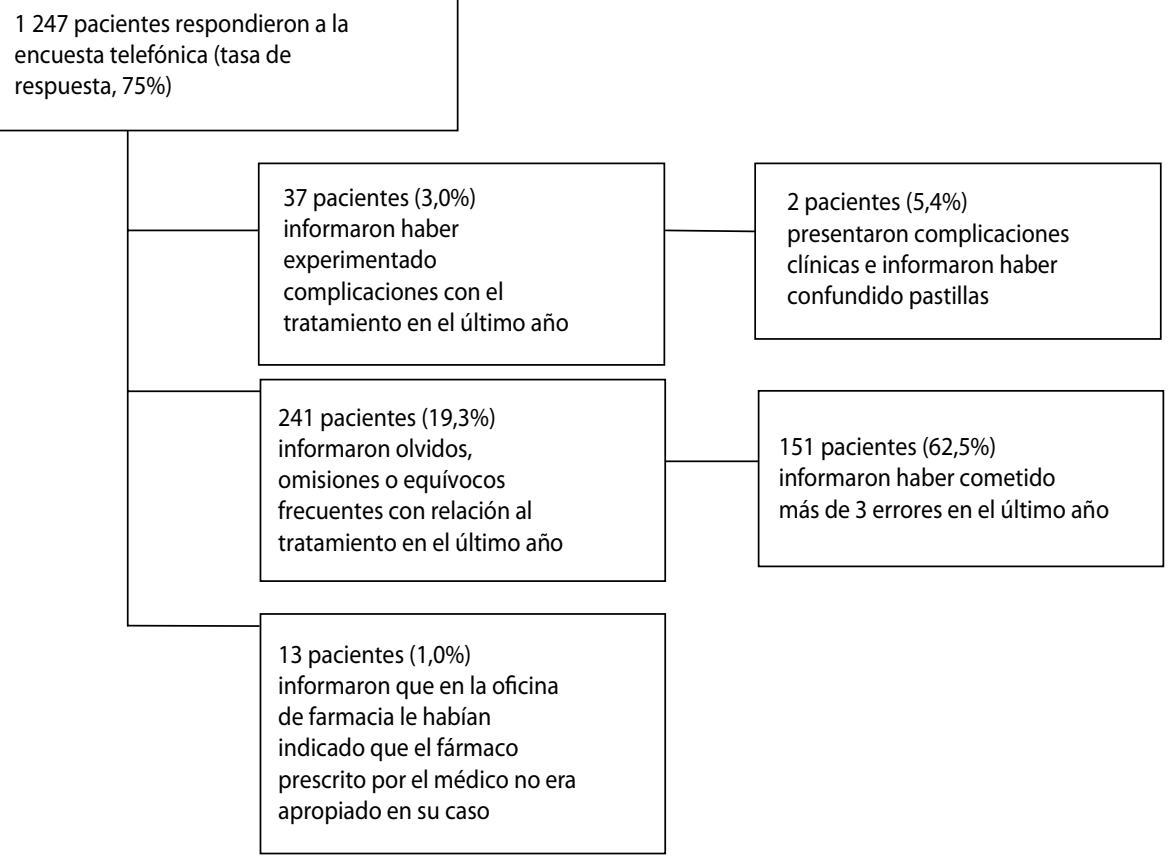

En 13 casos (1\%, IC 95\%: 0,5 a 1,6), al menos en una ocasión, en la oficina de farmacia se le había indicado al paciente que el tratamiento prescrito por su médico no era apropiado para él y le aconsejaron solicitar una nueva consulta con su médico (cuadro 1). La probabilidad de que el paciente informara esta indicación de la oficina de farmacia se incrementaba cuando en la encuesta el paciente valoraba negativa- mente la información recibida del médico, cuando disminuía su confianza en este o cuando tenía la sensación de que el tiempo de consulta no era el adecuado (cuadro 2).

Una valoración negativa de la información recibida por parte del médico, la sensación de quedarse con dudas sobre el tratamiento y la falta de confianza en su médico se asociaron a la frecuencia con que se informaron complicaciones en el curso del tratamiento (cuadro 3). No se hallaron efectos de la interacción entre las variables sexo y edad, lo que indica que la tendencia de los datos era la misma en todos los casos.

\section{DISCUSIÓN}

La frecuencia de EA debidos a deficiencias del sistema sanitario y errores involuntarios de los profesionales se ha descrito en varios estudios $(4,8,26)$. Sin embargo, la frecuencia de errores de los propios pacientes en la autoadministración de la medicación ha sido menos estudiada. En este estudio se destaca que una elevada proporción de pacientes (17 a $21 \%$ ) informan haber cometido errores relacionados con la medicación en su domicilio.

Los pacientes desempeñan un papel clave en la garantía de su seguridad cuando reciben cuidados sanitarios. En este estudio, los pacientes informaron complicaciones o reacciones inesperadas o indeseables asociadas a los tratamientos en una proporción similar a la observada en otros estudios, lo que sugiere 
CUADRO 2. Tiempo de consulta, valoración de la información proporcionada por el médico y confianza en el médico, y frecuencia con que en la oficina de farmacia alertan al paciente de un posible error de prescripción, Comunidad Valenciana, España, 2010

\begin{tabular}{lccc}
\hline & & \multicolumn{2}{c}{$\begin{array}{c}\text { Oficina farmacia } \\
\text { indica que el } \\
\text { tratamiento es } \\
\text { inadecuado }\end{array}$} \\
\cline { 3 - 4 } & & No. & $\%$ \\
\hline Tiempo de consulta $(n=1244)$ & Mal/Regular & 3 & $4,6^{\mathrm{a}}$ \\
& Bien & 10 & 0,9 \\
Información que le proporciona el médico & Mal/Regular & 5 & $5,5^{\mathrm{a}}$ \\
$(n=1239)$ & Bien & 8 & 0,7 \\
Confianza en el médico $(n=1242)$ & No & 3 & $4,1^{\mathrm{b}}$ \\
& Sí & 10 & 0,9 \\
\hline
\end{tabular}

Nota: se han agrupado las respuestas que denotaban una valoración negativa por parte del paciente.

a Prueba exacta de Fisher, $P=0,03$.

b Prueba exacta de Fisher, $P<0,002$.

CUADRO 3. Confianza y calidad percibida por el paciente de la información proporcionada por su médico, y complicaciones en el curso del tratamiento y frecuencia de errores del paciente, Comunidad Valenciana, España, 2010

\begin{tabular}{|c|c|c|c|c|c|c|c|c|c|}
\hline & & \multicolumn{2}{|c|}{$\begin{array}{c}\text { Efecto } \\
\text { inesperado o } \\
\text { complicación } \\
\text { en el último } \\
\text { año }\end{array}$} & \multicolumn{2}{|c|}{$\begin{array}{l}\text { Olvida con } \\
\text { frecuencia la } \\
\text { explicación } \\
\text { del médico }\end{array}$} & \multicolumn{2}{|c|}{$\begin{array}{l}\text { Olvida con } \\
\text { frecuencia } \\
\text { tomar la } \\
\text { medicación }\end{array}$} & \multicolumn{2}{|c|}{$\begin{array}{l}\text { Confunde con } \\
\text { frecuencia los } \\
\text { comprimidos }\end{array}$} \\
\hline & & No. & $\%$ & No. & $\%$ & No. & $\%$ & No. & $\%$ \\
\hline \multirow{2}{*}{$\begin{array}{l}\text { Sensación de ser escuchado } \\
\text { por su médico }(n=1236)\end{array}$} & Mal/Regular & 7 & 6,0 & 17 & 14,5 & 13 & 11,1 & 6 & 5,1 \\
\hline & Bien & 29 & 2,6 & 156 & 13,9 & 123 & 11,0 & 67 & 6,0 \\
\hline \multirow{2}{*}{$\begin{array}{l}\text { Se quedó con ganas de } \\
\text { preguntar dudas al médico } \\
(n=1243)\end{array}$} & Sí & 4 & 10,3 & 9 & 23,1 & 4 & 10,3 & 3 & 7,7 \\
\hline & $\mathrm{No}$ & 33 & $2,7^{\mathrm{a}}$ & 166 & 13,8 & 132 & 11,0 & 70 & 5,8 \\
\hline \multirow{2}{*}{$\begin{array}{l}\text { Confianza en el médico } \\
(n=1242)\end{array}$} & No & 6 & $7,9^{b}$ & 15 & 19,7 & 13 & 17,1 & 8 & 10,5 \\
\hline & Sí & 31 & 2,7 & 159 & 13,6 & 122 & 10,5 & 65 & 5,6 \\
\hline \multirow{2}{*}{$\begin{array}{l}\text { Tiempo de consulta } \\
\quad(n=1244)\end{array}$} & Mal/Regular & 5 & $7,5^{\mathrm{c}}$ & 10 & 14,9 & 9 & 13,4 & 4 & 6,0 \\
\hline & Bien & 32 & 2,7 & 165 & 14,0 & 127 & 10,8 & 59 & 5,9 \\
\hline \multirow{2}{*}{$\begin{array}{l}\text { Información que le } \\
\text { proporciona el médico } \\
(n=1239)\end{array}$} & Mal/Regular & 7 & $7,5^{d}$ & 14 & 15,1 & 10 & 10,8 & 3 & 3,2 \\
\hline & Bien & 30 & 2,6 & 158 & 13,8 & 123 & 10,7 & 67 & 5,8 \\
\hline
\end{tabular}

Nota: se han agrupado las respuestas que denotaban una valoración negativa por parte del paciente.

a $P<0,01$.

b $P<0,03$.

c Prueba exacta de Fisher, $P=0,04$.

d Prueba exacta de Fisher, $P=0,02$.

que pueden ser informadores útiles para mejorar la seguridad de los pacientes $(17,18)$, contribuyendo a la vez, si se los tiene en cuenta, al cambio necesario en la cultura de seguridad del paciente.

Sobre la base de una extrapolación de los datos del estudio APEAS (8) cabría esperar que presenten un EA cada año alrededor de $6 \%$ de los pacientes asistidos en las consultas de atención primaria, de los que casi la mitad se asocian a la medicación y una cuarta parte a problemas de comunicación entre profesionales y entre pacientes y profesionales. Este estudio apunta en la misma direc- ción, aunque aporta el dato hasta ahora no conocido del papel de las oficinas de farmacia en la cadena de la seguridad del paciente, identificando un posible error médico y advirtiendo al paciente que la prescripción del médico podría no ser apropiada en su caso. Cuando, en la encuesta, el paciente no valoró positivamente la información proporcionada por el médico o el tiempo de consulta que le ha dedicado, fue más frecuente que en la oficina de farmacia le indicaran que debía volver a su médico para consultar si la prescripción era apropiada en su caso, lo que confirma el riesgo asociado a una información deficitaria y a un tiempo de consulta limitado.

Según estos datos, el médico debe tener en cuenta que los pacientes también cometen errores que afectan a la seguridad clínica. Los pacientes son actores principales en el acto asistencial, y un miembro ocasional, pero esencial, del proceso de cuidados. Su papel en la seguridad clínica tiene más relevancia de la que se le atribuye habitualmente (27). En este estudio uno de cada cinco pacientes, de todas las edades, y especialmente los más jóvenes, tiene frecuentes olvidos, omisiones o equívocos con la medicación que, en algunos casos, requieren un nuevo tratamiento. Estos datos indican que debe prestarse atención a un factor escasamente considerado: los pacientes (o sus familiares o cuidadores) como causantes de EA.

Es muy probable que la atención primaria sea el área donde mejor puede afrontarse la neutralización de los riesgos asociados a los errores de los pacientes. Los datos de este estudio resaltan la importancia que tiene para la seguridad de los pacientes intervenir de forma activa en este nivel asistencial $(3,28)$. Si se tiene en cuenta que entre los pacientes crónicos y con patologías múltiples son más frecuentes los EA (29) (además, con un mayor número por paciente) parece aconsejable priorizar actuaciones en esta población de pacientes. En este sentido, los programas desarrollados al amparo del concepto del paciente competente podrían reorientarse, por ejemplo, haciendo partícipes a pacientes para ayudar a otros pacientes a identificar causas de posibles EA asociadas a olvidos, confusiones o equívocos frecuentes con la medicación, en especial si, como parece, los pacientes sobrevaloran la seguridad del tratamiento que siguen $(11,20)$.

Que el paciente juzgue como positiva la información que proporciona el médico se ha asociado a un menor número de complicaciones con la medicación, pero no a la frecuencia de errores de los pacientes. Lo mismo sucede con la duración de la consulta, en concordancia con los resultados de otros estudios $(24,30)$.

Kuzel y cols. (3) ya sugerían en 2004 que entre los problemas que afectaban a la seguridad se encontraban los relacionados con la información. Los datos más recientes de la Joint Commission on Accreditation of Health Organizations (31) confirman que este factor es una de las causas más frecuente de EA. 
No obstante, informar no parece ser suficiente (32). Que se haya informado al paciente no significa que este haya comprendido la información, ni que sea capaz de ponerla en práctica. Mejorar la comunicación verbal con los pacientes y sus familias podría contribuir a minimizar los errores clínicos (33); como parece deducirse de estos y otros resultados (30), esto requiere un tiempo mínimo de dedicación en la consulta.

Por otro lado, estos resultados destacan dos de las limitaciones que los pacientes encuentran para adoptar un rol más activo: el tiempo de consulta y la información. Es importante señalar que este rol se ha relacionado con su participación como segundo control para limitar las causas de los EA $(27,34)$.

En este estudio, la información proporcionada por el médico no se ha relacionado con la frecuencia con la que los pacientes informan de errores por acción u omisión en el ámbito de su hogar. Los encuestados no atribuyeron sus olvidos y confusiones ni a un tiempo insuficiente de consulta, ni a la falta de información. En cambio, los errores por acción u omisión de los pacientes sí se mostraron relacionados con la percepción de efectividad del tratamiento. Este resultado sugiere que estos errores causan también un mal aprovechamiento de los recursos sanitarios.

Recurrir a los pacientes como informadores tiene muchas ventajas, pero también inconvenientes. Por un lado se encuentra la dificultad de comprensión conceptual. Es complejo preguntar sobre la frecuencia de EA ya que, normalmente, estos se identifican como "errores médicos" $(25,35)$. Por otro, la dificultad para diferenciar entre un error y una reacción adversa a medicamentos (20). En este estudio se optó por preguntar sobre complicaciones y efectos inesperados en el curso del tratamiento que, en otros estudios (10), se habían identificado como indicativos de EA.
Este estudio tiene otras limitaciones. No sabemos por qué algunas personas se negaron a responder a la encuesta, aunque es probable que las razones estén ligadas al tedio que provoca el incremento de las acciones de marketing telefónico. Las informaciones proporcionadas por los pacientes durante las encuestas telefónicas no han sido contrastadas con datos clínicos. No obstante, hay datos que sugieren que las informaciones de los pacientes son razonablemente fiables (17). No se identificó el número de fármacos que consumía el paciente de manera simultánea, por lo que cabe sugerir, como línea de investigación en el futuro, el estudio de los errores de los pacientes polimedicados $y$ el análisis de los costes que suponen. Si bien el médico es una importante fuente de información para el paciente, no es la única. La confianza en el médico se determinó considerando la valoración del paciente de la atención recibida y si se planteaba un cambio de médico. No se consideró la información obtenida a través de Internet o de otros profesionales $\mathrm{u}$ otros pacientes. Estos resultados no pueden generalizarse a aquellos pacientes que han formalizado reclamaciones o demandas judiciales. Estos resultados no deben extrapolarse directamente a otros modelos sanitarios.

Las futuras investigaciones podrían ahondar en la descripción de los errores de los pacientes en la autoadministración de medicamentos y en la realización de controles de la medicación. Los profesionales disponen de información clínica relevante para aconsejar el mejor tratamiento a los pacientes, pero son estos quienes deben llevar a la práctica esas indicaciones. No podemos pensar que los pacientes son meros receptores de la información que se les proporciona. Para prevenir EA es probable que, más que la información que proporciona el médico de atención primaria, sea el estilo de comunicación y la comprensión del men- saje por el paciente lo que determina que este se involucre activamente en aras de su seguridad.

Dada la frecuencia de los errores de los pacientes, este estudio presenta el interrogante de su impacto en términos de daño, lesión o consumo de recursos, como se ha planteado en el caso de los profesionales. Asimismo, abre la puerta al estudio del papel de las asociaciones de pacientes para contribuir no sólo a reducir estos errores sino, incluso, a mitigar su impacto cuando no hayan podido evitarse.

Los pacientes, una vez en casa, tienen olvidos y cometen errores en la autoadministración de la medicación que ocasionan EA. La frecuencia de estos errores se incrementa cuando el paciente se manifiesta insatisfecho con la información que proporciona el médico. Las oficinas de farmacia, actuando como segundo control, contribuyen a fortalecer la cadena de la seguridad de los pacientes. Entre las necesidades de información de los pacientes crónicos cabe también incluir cómo evitar olvidos, confusiones o equívocos frecuentes con la medicación una vez en casa.

Agradecimientos. José Antonio Picó, Susana Lorenzo, Virtudes Pérez-Jover y Jesús Casal revisaron el borrador de la encuesta a pacientes. Lidia Ortiz colaboró en la confección de las bases de datos. Julián Vitaller revisó una primera versión de este original y contribuyó a su redacción. La revisión de experiencias y literatura se gestó gracias a una beca del Programa BEST/2010/219 de la Generalitat Valenciana que facilitó una estancia de JJM en la Organización Panamericana de la Salud con Dolors Monserrat y Cho Malhi. El estudio se realizó gracias a una ayuda del Fondo de Investigaciones Sanitarias, referencia PI08-90118, Instituto de Salud Carlos III, Ministerio de Ciencia y Tecnología de España.

\section{REFERENCIAS}

1. Gandhi TK, Weingart SN, Borus J, Seger AC, Peterson J, Burdick E, et al. Adverse drugs events in Ambulatory Care. N Engl J Med. 2003;348:1556-64.

2. Wilson T, Sheikh A. Enhancing public safety in primary care. BMJ. 2002;321:581-7.

3. Kuzel AJ, Woolf SH, Gilchrist WJ, Engel JD, La Veist TA, Vincent $C$, et al. Patient reports of preventable problems and harms in primary health-care. Ann Fam Med. 2004;2:333-40.

4. Ministerio Sanidad y Política Social. Estudio IBEAS. Prevalencia de efectos adversos en hospitales de Latinoamérica. Disponible en: http://www.msc.es/organizacion/sns/plan CalidadSNS/docs/INFORME_IBEAS.pdf Acceso el 28 de septiembre de 2010.
5. Borrell F, Páez C, Suñol R, Orrego C, Gil N, Martí M. Errores clínicos y eventos adversos: percepción de los médicos de atención primaria. Aten Primaria. 2006;38:25-32.

6. Garfield S, Barber N, Walley P, Willson A, Eliasson L. Quality of medication use in primary care - mapping the problem, working to a solution: a systematic review of 
the literature. BMC Medicine. 2009;7:50. doi:10.1186/1741-7015-7-50.

7. Rubin G, George A, Chinn DJ, Richardson C. Errors in general practice: development of an error classification and pilot study of a method for detecting errors. Qual Saf Health Care. 2003;12:443-7.

8. Aranaz JM, Aibar C, Vitaller J, Mira JJ, Orozco D. Estudio APEAS: estudio sobre la seguridad de los pacientes en Atención Primaria de Salud. Ministerio de Sanidad y Consumo (España). Madrid 2008. Disponible en: http:// www.msc.es/organizacion/sns/planCali dadSNS/docs/estudio_apeas.pdf Acceso el 28 de septiembre de 2010.

9. Awe C, Lin SJ. A Patient Empowerment Model to Prevent Medication Errors. J Med System. 2003;27:503-17.

10. EMERGE, the Erice Medication Errors Research Group. Medication errors: problems and recommendations from a consensus meeting. Br J Clin Pharmacol. 2009;67:592-8.

11. Mira J, Aranaz J, Vitaller J, Ziadi M, Lorenzo $\mathrm{S}$, Rebasa $\mathrm{P}$, et al. Percepción de seguridad clínica tras el alta hospitalaria. Med Clin (Barc). 2008;131(Supl 3):26-32.

12. Wilson RM, Runciman WB, Gibberd RW, Harrison BT, Newby L, Hamilton JD. The Quality in Australian Health care Study. Med J Aust. 1995;163:458-71.

13. Britten N. Medication errors: the role of the patient. Br J Clin Pharmacol. 2009;67:646-50

14. Einarson TR, Drug-related hospital admissions. Ann. Pharmacother.1993; 27:832-40.

15. Mira JJ. Pacientes por la seguridad de los pacientes. El papel del paciente en la seguridad de los pacientes. Med Preventiva. 2011;XVI:5-11.

16. Blendon RJ, Des Roches CM, Brodie M, Benson JM, Rosen AB, Schneider E, et al. Views of Practicing Physicians and the Public on Medical Errors. N Engl J Med. 2002;347:1933-40.

17. Metlay JP, Cohen A, Polsky D, Kimmel SE, Koppel R, Hennessy S. Medication Safety in Older Adults: Home-Based Practice Patterns. J Am Geriatr Soc. 2005;53:976-82.
18. Massó P, Aranaz J, Mira JJ, Perdiguero E, Aibar C. Adverse Events in hospitals: The patient's point of view. Qual Saf Health Care. 2010;19:144-7.

19. Weingart SN, Pagovich O, Sands DZ, Li JM, Aronson MD, Davis RB, et al. What can hospitalized patients tell us about adverse events? Learning from patient-reported incidents. J Gen Intern Med. 2005;20:830-6.

20. Mira JJ, Lorenzo S, Vitaller J, Ziadi M, Ortíz $\mathrm{L}$, Ignacio $\mathrm{E}$, et al. El punto de vista de los pacientes sobre la seguridad clínica de los hospitales. Validación del Cuestionario de Percepción de Seguridad. Rev Med Chile. 2009;137;1441-8.

21. European Commission. Patient safety and quality of healthcare. Special Eurobarometer. 327/Wave 72.2. Bruselas; abril de 2010.

22. Ministerio Sanidad y Consumo. Barómetro Sanitario. 2008. Disponible en: http://www. msc.es/estadEstudios/estadisticas/infor Recopilaciones/barometro/home.htm Acceso el 19 de septiembre de 2010.

23. Evans SM, Berry JG, Smith BJ, Esterman AJ. Consumer perceptions of safety in hospitals. BMC Public Health. 2006;6:41.

24. Northcott H, Vanderheyden L, Northcott J, Adair C, McBrien-Morrison Ch, Norton P, et al. Perceptions of preventable medical errors in Alberta, Canada. Int J Qual Health Care. 2008;20:115-22.

25. King A, Daniels J Lim J, Cochrane DD, Taylor A, Ansermino JM. Time to listen: a review of methods to solicit patient reports of adverse events. Qual Saf Health Care. 2010;19:148-57.

26. Aranaz-Andrés JM, Aibar-Remón C, VitallerBurillo J, Ruiz-López P, Limón-Ramírez R, Terol-García $\mathrm{E}$, et al. Incidence of adverse events (AEs) related to health care in Spain. Results of the Spanish National Study of Adverse Events (ENEAS). J Epidemiol Community Health. 2008;62:1022-9.

27. Longtin Y, Sax H, Leape L, Sheridan S, Donalson L, Pittet D. Patient participation:
Current knowledge and applicability to patient safety. Mayo Clin Proc. 2010;85:53-62.

28. Hickner J, Graham DG, Elder NC, Brandt E, Emsermann CB, Dovey S, et al. Testing process errors and their harms and consequences reported from family medicine practices: a study of the American Academy of Family Physicians National Research Network. Qual Saf Health Care. 2008;17:194-200.

29. Gea-Velázquez de Castro MT, Aranaz-Andrés JM. Eventos adversos asociados a la asistencia del paciente pluripatológico ingresado en hospitales de crónicos. Med Clín (Barc). 2010;135(Supl 1):17-23.

30. Mira JJ, Nebot C, Lorenzo S, Pérez-Jover V. Patient report on information given, consultation time and safety in primary care. Qual Saf Health Care. 2010;19.

31. JCOHA. 2010 National Patient Safety Goals (NPSGs). Disponible en: http://www.joint commission.org/patientsafety/national patientsafetygoals/ Acceso el 29 de octubre de 2010.

32. Barca I, Parejo R, Gutiérrez P, Fernández F, Alejandre G, López de Castro F. La información al paciente y su participación en la toma de decisiones clínicas. Aten Primaria. 2004;33:361-7.

33. Volpp KGM, Grande D. Patient Safety: Residents' Suggestions for Reducing Errors in Teaching Hospitals. N Engl J Med. 2003;348: 851-5.

34. Schwappach DL. Review: engaging patients as vigilant partners in safety: a systematic review. Med Care Res Rev. 2010;67:119-48.

35. Agoritsas T, Bovier PA, Perneger TV. Patient Reports of Undesirable Events During Hospitalization. J Gen Intern Med. 2005;20:922-8.

Manuscrito recibido el 17 de marzo de 2011. Aceptado para publicación, tras revisión, el 14 de junio de 2011.

\section{ANEXO I. Preguntas realizadas a los encuestados, Comunidad Valenciana, España, 2010}

En relación con la última vez que acudió a consulta con su médico, o este último año:

1. ¿Tiene la sensación de que el médico lo escuchó con suficiente interés y que tuvo en cuenta lo que usted le dijo?

2. ¿Se quedó con ganas de preguntar algo al médico? (Por ejemplo: algo que no hubiese entendido bien del todo, algo que no le explicó y que a usted le preocupara, etc.)

3. ¿Ha sufrido algún efecto inesperado o no deseado, o alguna complicación, con el tratamiento que estuviera tomando?

4. ¿Alguna vez en la farmacia le han dicho que ese no era el tratamiento más adecuado para usted?

5. Respecto de lo que usted esperaba no se ha sentido correctamente tratado y preferiría un cambio de médico.

Valore los siguientes aspectos:

6. La capacidad de su médico para comprender sus problemas de salud.

7. La dedicación del tiempo necesario en la consulta por parte de su médico.

8. La información que ha recibido de su médico.

9. En una escala de 0 a 10, donde 0 significa NADA SATISFACTORIO y 10 significa MUY

SATISFACTORIO. En general, ¿cuál fue su satisfacción global con las atenciones recibidas?

Indique con qué frecuencia le ha ocurrido en este último año:

10. Olvidar, una vez en casa, lo que el médico le ha dicho durante la consulta.

11. Olvidarse de tomar la medicación recetada por su médico.

12. Equivocarse o confundirse con las pastillas. 
ABSTRACT Objective. Analyze the frequency of medication errors committed and reported by patients.

Frequency of medication

Methods. Descriptive study based on a telephone survey of a random sample of errors by patients adult patients from the primary care level of the Spanish public health care system. A total of 1247 patients responded (75\% response rate); 63\% were women and $29 \%$ were older than 70 years.

Results. While 37 patients (3\%, 95\% CI: 2-4) experienced complications associated with medication in the course of treatment, $241(19.4 \%, 95 \% \mathrm{CI}: 17-21)$ reported having made some mistake with their medication. A shorter consultation time $(P<0.01)$ and a worse assessment of the information provided by the physician $(P<0.01)$ were associated with the fact that during pharmacy dispensing the patient was told that the prescribed treatment was not appropriate.

Conclusions. In addition to the known risks of an adverse event due to a health intervention resulting from a system or practitioner error, there are risks associated with patient errors in the self-administration of medication. Patients who were unsatisfied with the information provided by the physician reported a greater number of errors.

Key words Medication errors; medication adherence; patient participation; physician-patient relations; primary health care; Spain. 